\title{
Plutonium generates opposition
}

\section{Washington}

A COALITION of environmentalist and anti-nuclear groups is asking for a legal injunction to prevent the launch on 12 October of the space shuttle Atlantis, whose payload is the Jupiter-bound spacecraft Galileo. A lawsuit filed in the District of Columbia last Thursday claims that the National Aeronautics and Space Administration (NASA) has deliberately underestimated the chances of a launch accident which could release some of the $100 \mathrm{~kg}$ of plutonium that Galileo carries to generate power. But those behind the lawsuit would not say whether a reassessment of the risks could even in principle lead to an outcome they would find acceptable, and said that if legal efforts to stop the launch failed they would mount a programme of civil disobedience.

During Galileo's 22-month mission, it will study at close range both Jupiter and several of its satellites, and will drop a probe into the jovian atmosphere. To provide power for its many scientific instruments, it carries two radioisotope thermal generators (RTGs), each containing about $50 \mathrm{~kg}$ of plutonium in the form of ${ }^{238} \mathrm{PuO}_{2}$. The RTGs are not nuclear reactors; radioactive decay of the plutonium dioxide, made up into a number of 5 -cm-long fuel pellets sealed into iridium and carbon-boron epoxy-fibre capsules, releases heat which is converted into electric power by thermocouples. Plutonium is used because it emits mostly alpha-radiation, easily captured by the shielding and converted to heat, and little of the more penetrating and more dangerous gamma-radiation.

The lawsuit raises the possibility that if Atlantis, like Challenger almost four years ago, were to explode on launch, the RTGs could be blown apart, scattering plutonium powder across Florida. NASA, on the other hand, says that the RTGs have been physically tested, and shown to remain intact, under conditions more severe than a shuttle explosion.

The groups asking for the injunction are the Florida Coalition for Peace and Justice, an association of local groups active in human rights and anti-nuclear campaigning, the Washington-based Foundation for Economic Trends, led by Jeremy Rifkin, which has lobbied and litigated over many environmental issues, and the Christic Institute. The last of these is an idiosyncratic Washington organization with a history of involvement in antinuclear activities, such as the Karen Silkwood case and the radiation leak from the Three Mile Island reactor, but which is also known for its theory that US foreign policy, since the late $1950 \mathrm{~s}$, has been steered by a cabal of CIA (Central Intelligence Agency) and ex-CIA operatives of whom Oliver North is but the latest.

At a press conference to announce the filing of the lawsuit, Bruce Gagnon of the Florida Coalition, Rifkin and Lanny Sinkin of the Christic Institute quoted a variety of different numbers that they said NASA had used at different times for the probabilities of certain kinds of accident. Before the Challenger disaster, they said, the chance of a shuttle explosion had been put at one in 10 million; now it was as high as one in 35. NASA put the chance of plutonium release at one in 2,500 , but other official numbers given by Rifkin led to a figure of one in 430. Moreover, it was argued, NASA has suppressed risk assessments that disagreed markedly with its own, and has failed to include all relevant analyses in its Environmental Impact Statement for the Galileo mission.

The lawsuit thus argues that NASA has evaded its responsibilities under the 1982 National Environment Protection Act, and seeks to have the risk assessment analysis re-done, with all facts and figures made public. But, at the press conference, the ultimate purpose of this legal tactic was not clear. Neither Rifkin, Sinkin nor Gagnon would say what probability of plutonium release, determined by a new risk assessment, they would find acceptable. Sinkin likened an Environmental Impact Statement to a risk-benefit analysis; in this case, he suggested, the risk was clearly finite and the benefit, at least to the people of Florida, was nil.

Moving on to larger themes, Sinkin said that the "radioactivists" - his name for the Department of Energy (DoE), which supplies the plutonium to NASA - were bent on putting radioactive materials into space wherever they could. The purpose of this strategy, said Gagnon, was that by using civil missions such as Galileo to launch nuclear devices without arousing great public anxiety, the path would be cleared for the true ambition of DoE and the weapons industry, which was to put nuclear weapons into space.

Sinkin, Gagnon and Rifkin all argued that NASA's reliance on nuclear power for long-term missions far from the Sun could be changed if they were forbidden to use plutonium or other radioisotopes; they would be forced to find alternatives. It might take 10 or 15 years, Sinkin said, but Jupiter "would still be there".

The official response to the lawsuit, which names President Bush as well as NASA and DoE, will come through the Justice Department. A statement from NASA claimed that the press announcement of the lawsuit contained several "glaring inaccuracies", and a spokesman said that the reason that a variety of accident probabilities were in circulation was that there was no single way that plutonium might be released - an explosion on the launch pad and one five minutes into the flight would have quite different consequences, for example - and a suitable average of different probabilities had to be determined. For now, NASA is standing firmly by its estimate of 1 in 2,500 as the chance of a release of plutonium during the launch of Atlantis.

David Lindley

\section{Are video terminals dangerous?}

\section{Boston}

Almost four years after it was first proposed, the Mount Sinai School of Medicine in New York has begun a comprehensive, nationwide study intended to provide answers to long-standing questions about the reproductive risk to women officeworkers of constant exposure to video display terminals (VDTs). The four-year, \$2-million study began officially last month with funds from the National Institute of Child Health and Human Development.

Michele Marcus, the principal investigator, says the study "will examine the lifestyles, work activities, and health of 8,000 women office workers nationwide". Participants in four cities (New York, Boston, Cleveland and an as yet undetermined city in California) will be assessed by questionnaire, and 800 of the 8,000 will then be monitored closely for one-year during which time the women's menstrual cycles and hormone levels will be monitored, and their urine will be tested for human chorionic gonadotropin as a sensitive indicator of pregnancy. Provided that some of these 800 women become pregnant during the course of a year, the Mount Sinai study will be the first to monitor women office workers before pregnancy and throughout the first few months. Such a method seeks to control biases that have made questionable the results of some earlier studies. One such study, completed last year at the Kaiser Permanente Medical Center in California, found that women who used VDTs for over 20 hours per week had twice the miscarriage rate of those who did other work.

Louis Slesin, editor of the trade newsletter Microwave News, praises the Mount Sinai project and is happy to see the issue receiving federal support. Although the reality of the dangers of VDTs remains highly controversial, Slesin says that the Kaiser Permanente study "made the potential health risks credible", but adds that the Mount Sinai study, when it is completed in 1993, should provide a much fuller picture.

Seth Shuiman 